\title{
RELACIONES DE GÉNERO Y CONSTRUCCIÓN DE CULTURA DE PAZ EN LAS UNIVERSIDADES DE LA CIUDAD DE BOGOTÁ \\ Construyendo paz en las universidades con perspectiva de género
}

Gender Relations and Building a Culture of Peace in the Bogota's Universities: Building Peace in

Universities with a Gender Perspective

\author{
Angélica lucia Contreras Sierra ${ }^{1}$, Diana Marcela Ávila Fuentes ${ }^{2}$, Carlos Alberto Rubio Gallego ${ }^{1}$, \\ Leida Yamile Velásquez Camacho ${ }^{1}$, Laura Eunice Cuberos Obando ${ }^{1}$ \\ ${ }^{1}$ Corporación Universitaria Minuto de Dios, Colombia \\ ${ }^{2}$ Fundación Universitaria Monserrate, Colombia
}

\section{KEY WORDS}

Complexity

Culture of peace

Gender

Identity and education for

peace

Peace

Violence

\section{ABSTRACT}

This research highlights the studies carried out to determine how gender relations condition the construction of a culture of peace in higher education in the Bogota city, is developed with the participation of actors from three universities in the city. The study raises the perception that students, teachers and senior officials of these academic spaces have about the construction of the culture of peace in the universities of the city, with a differential approach from the gender perspective. This research is proposed from a hermeneutical phenomenological paradigm, with a qualitative and descriptive approach.
PALABRAS CLAVE

Complejidad

Cultura de paz

Género

Identidad y educación para la

paz

$\mathrm{Paz}$

Violencia

\section{RESUMEN}

género condicionan la construcción de cultura de paz en Educación superior en la ciudad de Bogotá, se desarrolla con la participación de actores de tres universidades de la ciudad. El estudio plantea la percepción que tienen estudiantes, maestros y directivos de estos espacios académicos, sobre la construcción de la cultura de paz en las universidades de la ciudad, con enfoque diferencial desde la perspectiva de género. Esta investigación se plantea desde un paradigma fenomenológico hermenéutico, con enfoque cualitativo y de tipo descriptivo. 


\section{Introducción}

$\mathrm{E}$ sta investigación emerge en el ámbito universitario, la pregunta que orienta la investigación es ¿Cómo influyen las relaciones de género en la construcción de la cultura de paz en las universidades (Universidad Pedagógica Nacional (UPN), Fundación Universitaria Monserrate (Unimonserrate) y Corporación Universitaria Minuto de Dios) de la ciudad de Bogotá? Las miradas que desde lo académico se dan en la comprensión de la cultura de paz, y las acciones que la evidencian, son el foco de esta investigación, buscando proponer desde este entorno, factores de asertividad, coherencia y corresponsabilidad, que sirvan de guía a las relaciones que desde allí se construyen, para favorecer la participación entre quienes se busca la igualdad de condiciones: mujeres y hombres, transformando así, la calidad en los entornos universitarios y propiciando el fortalecimiento de la cultura de paz.

La base de la aplicación de la propuesta de investigación se da con la participación de tres Instituciones Educativas de Educación Superior: Fundación Universitaria Monserrate (Unimonserrate), Universidad Pedagógica Nacional (UPN) y Corporación Universitaria Minuto de Dios, partiendo de una comunidad educativa: estudiantes de pregrado, docentes y directivos en formación académica posgradual de programas de educación y ciencias humanas, desde quienes se pueden observar dinámicas sociales en la construcción de una cultura de paz en los ámbitos universitarios, con un lente diferenciador, la perspectiva de género.

Este proyecto investigativo, se desprende de la macro-investigación institucional titulada "Cultura de Paz en los Programas de Educación y Ciencias humanas y sociales de las instituciones de educación superior de la ciudad de Bogotá", proyecto desarrollado a partir del segundo semestre del año 2016, adelantado por el grupo de investigación de la Fundación Universitaria Monserrate UNIMONSERRATE, llamado "Procesos sociopolíticos contemporáneos".

Se establece como objetivo general de la investigación, identificar cómo las relaciones de género condicionan en la construcción de la cultura de paz en las universidades de Bogotá, tomando como referencia los agentes educativos que allí se convocan. Como objetivos específicos en primer lugar se plantea caracterizar las percepciones de estudiantes, sobre el tema del condicionamiento de las relaciones de género en la construcción de cultura de paz en las universidades de Bogotá. En segunda medida, construir reflexiones desde una perspectiva educativa, pedagógica y hermenéutica sobre lo que conciben estudiantes, docentes y directivos sobre cultura de paz, relaciones de género y el papel de las universidades en la formación y el fortalecimiento de valores que fomenten la paz, en la ciudad de Bogotá. Finalmente, busca identificar la importancia del tema de género y las relaciones en un contexto de formación superior.

Cabe resaltar que al pensar en las relaciones construidas en algunas de las universidades como las ya mencionadas, es importante comprender las diversas dinámicas que se viven en su composición social, respecto a la multiculturalidad que allí emerge, teniendo en cuenta que Bogotá es la capital de la pluralidad, en cuanto a grupos étnicos, poblaciones víctimas de conflicto armado, que llegan de todas partes del país, personas en condición de discapacidad e incluso el género tomando en cuenta a las comunidades de los sectores LGBTIQ+ (Lesbianas, gay, bisexual, transgénero, transexual, travesti, intersexual y queer) quienes se movilizan para abrir espacios de formación que les posibiliten oportunidades de desarrollo y de equidad. Los ámbitos universitarios, son escenarios que nos invitan a desarrollar este tipo de estudios, desde diferentes posturas políticas, religiosas y sociales, comprendiendo que desde allí se podrán continuar gestando proyectos para la construcción de una paz positiva.

Esta investigación se enfocó en realizar un estudio, que diera cuenta y resaltara la importancia del papel que tienen las relaciones de género, dentro del desarrollo de la Cultura de Paz, haciendo visible la multiplicidad $y$ diversidad de miradas y saberes, desde donde se abre el espectro de posibilidades, a cambios culturales, resignificando dichas relaciones, basándolas en la protección, el respeto de los derechos desde el reconocimiento de las particularidades que nos hacen diferentes y a la vez sujetos partícipes y constructores de 
sociedades garantes de derechos y promotores de igualdad.

También es importante, identificar las acciones que algunas universidades implementan en su contexto, con el propósito de contribuir a la construcción de la cultura de paz desde los entornos académicos, fomentando una educación para la paz, como aspecto fundamental para la vida, comprendiendo la educación como una herramienta emancipadora, que fomenta $y$ encamina el desarrollo, hacia la equidad de oportunidades, poniendo en equilibrio las capacidades de hombres y mujeres en la construcción de sociedades más justas y pacíficas.

Es importante reflexionar que al hablar de paz, en la actualidad, hablamos no solo de un asunto netamente político, sino que también, la sociedad en general ha creado conciencia acerca de la responsabilidad social que implica la paz sobre un enfoque de corresponsabilidad, en este caso a la luz del papel que juegan las universidades como escuelas de educación superior, en el fortalecimiento de las sociedades y su desarrollo integral, reflexión que sin duda, se hace más compleja desde la perspectiva de género.

\section{Hilos conceptuales}

Teniendo en cuenta, el curso de la investigación y las relevancias que surgen es importante definir los siguientes conceptos que permiten vislumbrar con mayor claridad, los datos arrojados y analizados para comprender las percepciones y las propuestas de los ciudadanos, que se forman y hacen parte de las universidades participantes.

La paz es uno de los términos más polisémicos que existen. Aunque desde diferentes ambientes, situaciones y circunstancias se utilice la palabra paz, esta expresión no significa para todos lo mismo.

Uno de los mayores exponentes de los estudios sobre la paz, es el noruego Johan Galtung, quien plantea que el problema está, en que no es posible pensar la paz en un sentido único. Es decir, "es mejor pensar la paz en plural, como paces" (Jiménez, 2012, p. 6). Esta expresión nos permite replantear el concepto a nivel cultural, partiendo de una nueva propuesta para la construcción del sentido de paz, desde las reflexiones de la multiplicidad de formas de construirla. Algunos tipos de paz responden a definiciones como la paz negativa, paz positiva, paz positiva directa, paz positiva estructural, paz positiva cultural y la paz imperfecta.

Según Yudkin (2014), la paz negativa tiene su eje central en la cultura greco-romana. Este concepto comprende la paz como la ausencia de guerra, vista como guerras entre estados, siendo este el término más asociado por la sociedad. Por otra parte, la paz positiva surge en contraposición de la paz negativa, puesto que critica que al no reconocer las consecuencias del conflicto (otras formas de violencia distintas a la guerra), se obstaculiza el reconocimiento de la legitimación de una forma distinta de construir la paz.

...en la visión positiva de la paz se adopta una posición activa de construcción de paz al buscar reducir la violencia estructural o indirecta, luchando por los derechos humanos, la justicia social y la equidad. Si la paz se construye supone nuestra participación en el proceso de construirla. Cuando educamos para la paz y la convivencia, asumimos la responsabilidad de generar espacios de construcción de paz y de reducción de la violencia en todas sus manifestaciones, la violencia directa de la agresión y la violencia indirecta que genera vulneración de derechos, y por lo tanto, injusticia y exclusión. (Yudkin, 2014, p. 23)

Por otro lado, la paz directa se refiere a todas las necesidades básicas de los seres humanos (supervivencia, bienestar, libertad e identidad), direccionadas a la bondad verbal y física donde el bien para el cuerpo, la mente y el espíritu confluyen en el bienestar propio y del otro. "Allí el amor es entendido como la unión de cuerpos, mentes y espíritus" (Jiménez, 2012, p. 27).

La paz positiva estructural considera la importancia de sustituir "represión por libertad, equidad por explotación, integración en lugar de segmentación, solidaridad en lugar de fragmentación y participación en lugar de marginación". Aquí la necesidad es lograr la armonía del cuerpo, la mente y el espíritu. La clave en este caso es el diálogo interno y externo con uno mismo (Jiménez, 2012, p. 28). Desde el concepto anterior se da relevancia al concepto de paz positiva cultural en la cual la clave es el diálogo interno con uno mismo, lo que significa abrirse a diversas inclinaciones $y$ aptitudes 
humanas, sin reprimirlas, lo que sustituye la legitimación de la violencia por la legitimación de la paz, construyendo una cultura de paz positiva (Jiménez, 2012, p. 28).

Finalmente, en el amplio espectro conceptual de paz surge la paz imperfecta, siendo "el adjetivo imperfecto sirve para abrir, en algún sentido los significados de la paz. Aunque etimológicamente puede ser entendido como inacabada, procesual, y este es el significado central." (Muñoz, 2006), lo que reconoce la amplitud de consideraciones de una paz que se construye permanentemente.

Desde la comprensión de las paces, la Cultura de Paz se basa históricamente en la evolución de los sucesos que acontecen y convocan a la emergencia de acciones para mejorar las condiciones de las personas en los territorios, es así que, para las Naciones Unidas, durante la finalización de la segunda guerra mundial, toman importancia el estudio de la paz y la cultura de paz, tras las múltiples masacres y vulneración de los derechos humanos; es por esto que, en el siglo $\mathrm{XX}$, estos temas toman mayor relevancia, como un instrumento de gestión $y$ transformación de las entidades humanas (Muñoz \& Molina, 2010). En el año 1999 en la Declaración y Programa sobre una Cultura de Paz se establece con mayor puntualidad que la cultura de paz

es un conjunto de valores, actitudes, tradiciones, comportamientos y estilos de vida, que llevan implícitos el respeto a la vida, el fin de la violencia y la promoción y práctica de la no violencia por medio de la educación, el dialogo y la cooperación. (Muñoz \& Molina, 2010, p. 3)

Entonces, la cultura de paz implica los esfuerzos para satisfacer las necesidades de desarrollo y protección del medio ambiente; el fomento a la igualdad y a la equidad de derechos entre mujeres y hombres, la promoción de la libertad de expresión, opinión e información, entre otras consideraciones.

Desde diferentes miradas la definición de cultura de paz ha evolucionado en la misma medida en que han avanzado los conceptos de Paz y Cultura. Muñoz y Molina (2010) desagregan los términos para conceptualizarlos desde su raíz etimológica, concluyendo de esta forma que la Cultura de Paz va más allá de la simplificación puntual por definición de sus términos puesto que esta, es tan compleja, como lo son las relaciones humanas entre sí y con los entornos asociados a la interacción diaria.

De allí la importancia de poder educar para la paz, facilitando la transformación de los conflictos, fomentando relaciones participativas y coherentes desde una corresponsabilidad, con nuestra democracia y nuestra sociedad, respetuosa desde los derechos humanos con la aceptación de la diversidad como diferenciador potencial de construcción.

Es por esta razón que exaltamos uno de los objetivos de la cultura de paz, teniendo en cuenta el factor corresponsable que nos involucra a todos en la construcción de esta cultura, en la que "terminar con esa fascinación que el sexo masculino siente por la violencia es uno de los grandes retos que tiene, no sólo la educación para la paz, sino la misma convivencia humana, $y$ es un factor esencial, sino el más importante, de la cultura de paz" (Fisas, 1998, p. 4).

Para comprender con mayor claridad la importancia de construir la cultura de paz en los diversos contextos sociales, es necesario retomar las concepciones de la violencia, esto con miras a determinar con claridad cuáles son los aspectos que debemos romper como estereotipos culturales y estructurales de las relaciones, en el mundo en el que nos desenvolvemos. La violencia ha sido una constante en la humanidad, desde la historia antigua hasta la moderna vemos la descripción de guerras, brotes de violencia y acciones de poderío de unos sobre otros, los "más fuertes sobre los débiles", incluso en la literatura, el arte, la música se hacen visibles los actos violentos de las diferentes culturas alrededor del mundo, cuya dialéctica se lee, violenta en la mayoría de las situaciones conflictivas. La violencia se concibe como la coacción física o psíquica ejercida sobre una persona para viciar su voluntad y obligarla a ejecutar un acto determinado; puede adoptar distintas formas como física, verbal, psíquica, sexual, social, económica entre otras. (Expósito, 2011).

Se considera que muchas de las violencias, son ejercidas porque social y culturalmente, han sido aprobadas o porque las personas no están 
preparadas para solucionar los conflictos a los que se enfrentan, en distintos aspectos de la vida, como menciona Fisas citando al pedagogo Bruno Bettelheim, "la violencia es el comportamiento de alguien incapaz de imaginar otra solución a un problema que le atormenta" (Fisas, 1998, p. 18); por otra parte la Organización Mundial de la Salud establece algunos tipos de violencia como la dirigida contra uno mismo, la violencia interpersonal, la intrafamiliar, la violencia comunitaria, la violencia colectiva y la violencia de género. Sin embargo, estos tipos de violencia son más puntuales y existen unas generales, que sin duda son importantes conocer.

Para esta exposición se retoma el concepto de violencia cultural, considerada como aquella que legitima la violencia directa y la estructural a nivel social y de grupos sociales, esta violencia está dada por todos aquellos discursos, símbolos, metáforas, representaciones, himnos patrióticos o religiosos. Es importante entonces aclarar que la violencia directa es aquella que se da cuando un agente $o$ un actor intencionalmente busca hacer daño en el cuerpo y la mente de otro; y la violencia estructural o indirecta se define como la inserta en los espacios personales, sociales y mundiales, y no es intencionada.

En cuanto a esta violencia Johan Galtung introduce este concepto como parte de una comprensión de la paz como ausencia de violencia, (Jiménez, 2012, p. 26). De un modo genérico afirma que la violencia está presente,

cuando los seres humanos se ven influidos de tal manera que sus realizaciones efectivas, somáticas y mentales, están por debajo de sus realizaciones potenciales. La violencia es entonces la causa de la diferencia entre lo potencial y lo efectivo, aquello que aumenta su distancia y lo que obstaculiza su decrecimiento. (Jiménez, 2012, p. 26)

Teniendo en cuenta los conceptos anteriormente compartidos en donde se expresa la paz positiva como el ideal desde la construcción colectiva, social y democrática surge entonces la necesidad de comprender algunos otros enfoques que cobran relevancia en el camino de la Cultura de Paz como el concepto de género, el cual se concibe como las representaciones que se le asignan a las personas desde diferentes aspectos que cultural e históricamente se establecen, sin embargo, el género es una construcción también personal desde los sentires propios del individuo.

Es el conjunto de ideas, representaciones, prácticas y prescripciones sociales que una cultura desarrolla desde la diferencia anatómica entre mujeres y hombres, para simbolizar y construir socialmente lo que es "propio" de los hombres (lo masculino) y "propio" de las mujeres (lo femenino). (Lamas, 2000, p. 2)

Bastidas y Hernández citando a Joan Scott afirman que: "el género es un elemento constitutivo de las relaciones sociales basadas en las diferencias que distinguen los sexos y es una forma primaria de relaciones significantes de poder" (Bastidas \& Hernández, 2008, pág. 80).

Entonces, teniendo en cuenta la visibilización del concepto de violencia cultural como precedente y la concepción del género, es necesario identificar lo que se comprende por la violencia de género y desde allí concientizarnos sobre este espectro arraigado culturalmente en la sociedad para desde esa base consolidar acciones de cambio, personales y colectivas. Así pues la violencia de género desde la ONU como "todo acto de violencia sexista que tiene como resultado posible o real un daño físico, sexual o psíquico, incluidas las amenazas, la coerción o la privación arbitraria de libertad ya sea que ocurra en la vida pública o en la vida privada" (Expósito, 2011, p. 20). Francisca Expósito experta en el tema, asegura que la asimetría social en las relaciones entre mujeres y hombres favorece la reproducción de esta forma de violencia. La autora en mención asegura también que la violencia ha adquirido con el tiempo un significado social, tergiversando su significado original y creando el binomio violencia y género; se ha convertido en una conducta instrumental que introduce desigualdad en una relación interpersonal o mantiene una desigualdad subyacente y estructural (Expósito, 2011, pág. 20).

La identidad también tiene un papel importante en este estudio puesto que esta responde a una necesidad imperante de pertenencia, que permite la construcción propia y personal, que hace a los individuos ser parte de un sistema y a la vez sujeto de singularidad; en 
este sentido y teniendo en cuenta la perspectiva de género de nuestra investigación, resaltamos la postura de Marcela Lagarde quien afirma que:

La identidad de las mujeres es el conjunto de características sociales, corporales y subjetivas que las caracterizan de manera real y simbólica de acuerdo con la vida vivida. La experiencia particular está determinada por las condiciones de vida que incluyen, además, la perspectiva ideológica a partir de la cual cada mujer tiene conciencia de sí y del mundo, de los límites de su persona y de los límites de su conocimiento, de su sabiduría, y de los confines de su universo. (Lagarde, 1990, pág.1)

Nuestra sociedad evidencia que la masculinidad también ha sufrido cambios significativos y transforma las relaciones en la medida en que las dinámicas del género también se modifican.

A modo de articular todos los conceptos presentados, la Educación para la paz se concibe como la estrategia para la construcción de acciones, que mitiguen las problemáticas sobre violencias en los diferentes entornos académicos y sociales, que a su vez abren la mirada hacia la igualdad y la equidad de géneros. Suarez refiere a Morín (1999) atendiendo al concepto de educación para la paz estableciendo al respecto que "los individuos conocen, piensan y actúan según los paradigmas inscritos culturalmente en ellos" (Suarez, 2011). Morín también habla del "imprinting cultural" que nos marca con una cultura familiar, escolar y de desempeño escolar $\mathrm{y}$ nos hace ser quienes somos y cómo reaccionamos. "En la actualidad se hace necesario definir una educación en valores a la luz de nuevos paradigmas, como el enfoque complejo." (Suarez, 2011, p. 71), por esto la importancia de comprender que la educación para la paz es un tema que se debe trabajar en todos los ámbitos, desde lo familiar, la escuela y demás entornos de interacción de las personas, puesto que, en las tramas que se entretejen surgen los cambios que van impactando en cadena, las relaciones.

En atención a lo expuesto anteriormente, se centra la mirada en la universidad desde donde se deben promover propuestas, que permitan dar línea a un desarrollo positivo y activo de relaciones, basadas en la tolerancia, el amor, el respeto, reconocimiento e igualdad como parte de la construcción de la cultura de paz, en la que no se niegue el conflicto como parte del ser pero, a su vez, se brinden las herramientas para la resolución pacífica de estos, rompiendo con el paradigma de la violencia como mecanismo de mediación.

Finalmente y con el ánimo de vislumbrar la multiplicidad de constelaciones en cuanto a las relaciones que se construyen en este caso en los ámbitos universitarios, la complejidad permite el análisis del proceso investigativo toda vez que esta busca comprender sistemas abiertos, sistemas vivos, es necesario comprender desde la complejidad, las implicaciones que surgen en las relaciones de género emergentes dentro de las universidades, para este caso, que determinan las dinámicas sociales que replican los estereotipos de violencia o que por el contrario abren nuevas oportunidades para relacionarse, basados en valores y conductas que promueven la construcción de paz, impactando a su vez en otros entornos (sistemas) fuera de las universidades, como las familias y los espacios laborales y sociales.

Es importante, al hablar de complejidad en este artículo, dialogar sobre el ser humano como parte fundamental de los procesos aquí expuestos; las personas poseemos una naturaleza compleja en su totalidad, no solo desde la biología sino desde la comunicación, la interacción con el medio y con los otros, por esta razón es importante tener una mirada integral, que no fragmente a los individuos sino que los vea en su conjunto, una mirada que analice las dinámicas que se entretejen en unos y otros y la construcción que desde estas redes se dan, lo que permite asumir la complejidad como un factor conformador de vida y dinamismo, transformador de pensamiento y conocimiento el cual afecta de maneras directas o indirectas nuestro actuar y las lógicas sobre las cuales estamos fundamentados, lo que nos obliga a la reconfiguración de conceptos y la resignificación de experiencias y construcciones. En la perspectiva de María Cárdenas y José Rivas la teoría de la complejidad "surge como un paradigma científico cuya finalidad es 'comprender la complejidad de la vida'. Este paradigma ofrece el basamento teórico necesario 
para estudiar objetos muy complejos, como la escuela, sin la necesidad de proceder a una reducción radical de los mismos." (María L. Cárdenas R.; José F. Rivas R, 2004, pág. 131)

\section{Metodología}

Se planteó desde un paradigma fenomenológico hermenéutico, con enfoque cualitativo y de tipo descriptivo. Se consideró este tipo de investigación, puesto que se describieron las informaciones brindadas por cada uno de los agentes socioculturales de las tres universidades que hacen parte del estudio.

En la investigación se realizaron 16 entrevistas semiestructuradas a estudiantes, docentes y directivos de las universidades ya mencionadas anteriormente; en las que se indagó principalmente, los conocimientos que tenían estos actores sociales, sobre el tema de género, cultura de paz, y cómo evidenciaban las relaciones desde diferentes puntos de vista y las acciones que se realizaban desde las universidades en la construcción de una cultura de paz y a su vez se llevó, desde este proceso de entrevista, a la reflexión desde sus propios aportes en la construcción de cultura de paz, basados en el principio de corresponsabilidad.

Para el desarrollo de la investigación, se plantearon dos etapas, cada una con una duración de un año (12 meses); la primera definida como la aproximación, trabajo de campo y recolección de la información, y la segunda, análisis, interpretación y resultados finales.

En la primera etapa, se buscó realizar un reconocimiento y exploración en los escenarios en donde se iba a desarrollar la investigación (Universidad Pedagógica Nacional (UPN), Fundación Universitaria Monserrate (Unimonserrate) y la Corporación Universitaria Minuto de Dios), con los agentes de la comunidad educativa de acuerdo con los criterios a) de pertinencia: agentes socio-culturales que tuvieran alguna relación e interacción en la actualidad con la universidad, el género y las relaciones que allí emergen y b) la disponibilidad: además que los estudiantes, docentes y directivos mostraran interés por participar en el proceso.

Una vez finalizado este proceso, se trabajó en el diseño de instrumentos según el paradigma epistemológico de la investigación y las técnicas de recolección de información seleccionadas, a su vez, se realizó la planeación del trabajo de campo de acuerdo con el cronograma establecido previamente por las investigadoras. Finalmente, este primer momento terminó con la recolección de la información y posterior transcripción y sistematización.

Se aplicó la entrevista semiestructurada como instrumento de investigación, que como menciona Martínez (1998) es un instrumento técnico en donde los discursos se provocan a partir de una línea de trabajo flexible establecida en un marco situacional y conversacional, caracterizado por la circularidad que puede darse en la información que entregan los entrevistados y provocar al entrevistado. Los datos que se obtienen son de orden empírico y es allí donde el investigador puede tomar la decisión de respetar el lenguaje de los entrevistados y cuidar que sus expresiones no obstaculicen los significados que les asignan estas personas, estos resultados representan información útil para la reflexión y reconsideración de las situaciones planteadas en la investigación, realizando un análisis cualitativo que aporta a la investigación.

En la segunda etapa, se hace referencia al proceso que posibilitó realizar un diálogo de intersubjetividades, pasando de un plano descriptivo a uno interpretativo (Ruiz, 2006) de las subjetividades de los agentes socioculturales y los investigadores.

Para que fuera posible realizar los análisis de datos del presente proyecto de investigación, se utilizó el software Atlas.ti, el cual permitió el registro de acuerdo con los avances, las variables y demás datos cualitativos. Se aplican en conjunto la herramienta computacional y la interpretación de los investigadores buscando determinar inferencias y conclusiones frente a la problemática investigada.

\section{Resultados}

Tres familias de categorías surgen del proceso de recolección de información, por medio de las entrevistas semiestructuradas, mencionadas anteriormente; estas entrevistas se aplicaron a algunos actores de la comunidad educativa: estudiantes, docentes y directivos de las 
universidades que participaron en la investigación. Las categorías se definieron teniendo en cuenta los fundamentos teóricos de los temas de investigación y a su vez lo que los entrevistados expresaron. Las categorías son: CATEGORIA 1 Concepciones de género y cultura de paz de los miembros de la comunidad educativa, CATEGORIA 2 Relaciones que se construyen dentro y fuera de los espacios de formación que permean, refuerzan o resignifican los roles de hombres y mujeres en sus comunidades y CATEGORIA 3 Acciones que desde la corresponsabilidad se realizan con el fin de construir una cultura de paz desde las relaciones de género en los entornos académicos universitarios.

Para la categoría 1 encontramos que, para los jóvenes estudiantes de las universidades participantes, el concepto de cultura de paz es claro y puntual, tal y como lo expresa uno de los entrevistados: "refiere a la agrupación de actitudes y aptitudes que permitan generar ambientes de tolerancia, aceptación por el otro y solidaridad en todas las circunstancias, es decir se evidencian una suma de acciones, propuestas, construcciones y deconstrucciones que nutran la solidaridad y el respeto de manera individual y colectiva, permitiendo espacios de común acuerdo, contextos armoniosos, mediante el diálogo como primer herramienta de resolución de conflictos" (estudiante entrevistado, 2016), sin embargo son también, conscientes de las dificultades que se presentan para la construcción de esta paz, puesto que la cultura de la violencia está más presente en los entornos y las acciones que no son coherentes y no permiten fundamentar otras acciones para entablar la paz como estilo de vida, son acciones para nada consecuentes, ni coherentes y más bien se quedan en los discursos.

Las concepciones que se plantean docentes y estudiantes frente a la Cultura de Paz, entretejen una realidad que evoluciona, en la medida en la que somos más conscientes de nuestra responsabilidad en la construcción de una sociedad más justa e igualitaria que pretende la equidad, por lo que resaltamos el relato de uno de los entrevistados docente, quien afirma que es importante "entender que somos una sociedad construida desde la diversidad y en donde las variantes dinámicas de cada ser humano son la base fundamental para una convivencia, pensando en una construcción colectiva generadora de acciones en un territorio global e individual mediando por soluciones dadas desde el dialogo que nos permite reconocernos desde los derechos y deberes que nos constituyen como agentes sociopolíticas en los entornos en los que nos desenvolvemos" (Docente entrevistado 1, 2016)

El concepto de género aún es tergiversado y no muy comprendido por los estudiantes universitarios, esto se evidencia desde los discursos y los relatos que se dan en los espacios académicos, también es evidente que frente a este tema la universidad debe fortalecer $y$ ahondar en estos nuevos conceptos, que hacen parte de la construcción de una sociedad equitativa, que procure orientarse hacia la cultura de paz, por lo que hay un llamado a abordar con mayor fuerza el tema de la perspectiva de género en la comprensión de las realidades sociales, al igual que necesita realizar una ruptura clara y unánime de los paradigmas establecidos, en cuanto al ser hombre y mujer y los roles desde lo femenino y lo masculino, que se fundamentan a nivel social, es importante que los docentes en sus construcciones permitan a sus estudiantes reflexionar con lentes de género las relaciones existentes y como menciona un maestro entrevistado permitir "la comprensión que culturalmente la sociedad se ha definido en conceptos idealizados obviando lo aceptado y lo vivido de la diversidad y la inclusión, lo cual nos lleva a establecer roles muy limitados en el desenvolvimiento de hombres y mujeres"(docente entrevistado 2, 2016).

La concepción del género se da entonces, desde las relaciones problemáticas que no evidencian una plataforma de justicia transversal de equidad de género, cuando se habla de la visión que se da entre hombres y mujeres y el lugar que ocupan estas, en la construcción de cultura de paz, desde la voz de cada uno de los entrevistados, se evidencia la necesidad de sugerir, proponer, dialogar desde el lugar de sujetos políticos participes y activos de una sociedad y un contexto, en este caso las universidades; una necesidad de implementar propuestas en pro de favorecer la igualdad y el reconocimiento con propósitos de generar 
armonía, desarrollo y calidad educativa. Es importante resaltar la trascendencia de que la igualdad entre hombres y mujeres impacta desde el tema social y se evidencia como necesidad para construir cultura de paz con prospectiva económica y educativa, características esenciales en una sociedad.

En la categoría 2 analizamos que son varios los aspectos, desde el punto de vista de las relaciones entre hombres y mujeres, en la interacción que se da en la cotidianidad, no solo desde las universidades, sus aulas y otros entornos en los que confluyen hombres y mujeres con diferentes roles en la comunidad educativa, sino también fuera del alma mater que terminan influenciando las relaciones en cada entorno, en que se desenvuelven las personas.

A partir de sus vivencias en los entornos universitarios, indagamos sobre cómo consideran que son los lazos de cooperatividad entre hombres y mujeres, aspectos de competitividad entre ellos $\mathrm{y}$ ellas en lo académico; también se analizan, la diversidad de géneros y las concepciones que tienen los actores frente a la masculinidad y la feminidad.

Para los docentes la construcción del género y las identidades, permean las relaciones entre mujeres y hombres en la universidad y fuera de ella, uno de los entrevistados expresa que: "hay que hablar de feminidades y masculinidades en plural, pues se trata de las variaciones históricas que han sufrido los roles de género, porque en cada época y en las diversas culturas, esos papeles asignados a hombres y mujeres no son estáticos sino diversos, no son determinados como una especie de universales biológicos que diferencien para siempre a los hombres y a las mujeres. Los estudios históricos, antropológicos y sociales en general han establecido que a finales de la década de los años 60 esos patrones de masculinidad y feminidad sufrieron una gran alteración por efecto del ingreso masivo de las mujeres al trabajo y al estudio, esta tendencia generalizada en las sociedades contemporáneas alteró las prácticas de nupcialidad, las maneras de paternar y maternar con los hijos, incluso los desarrollos científicotécnicos como la popularización de la píldora y otros métodos anticonceptivos, y la fertilización in vitro, introdujeron una mayor liberalidad sexual en la cultura, también las distintas fases del movimiento feminista en su lucha contra el patriarcalismo influyeron en que el proyecto de vida de las mujeres sobre todo, ya no sea casarse y tener hijos, así mismo las luchas identitarias por el reconocimiento de diversos tipos de orientación sexual y el progreso hacia el reconocimiento de los derechos de las homosexualidades (gays, lesbianismos, intersexualidades, trasnsexualidades, metrosexualidades...) ponen de presente que las feminidades y masculinidades ya no son lo que antes eran y en un futuro próximo no serán tampoco lo que ahora son"(docente entrevistado 3, 2016). Teniendo en cuenta lo expresado por uno de los docentes entrevistados, evidenciamos cómo los grupos sociales, considerados minoritarios, han ido abriendo poco a poco espacios en ámbitos como la academia, buscando garantizar su participación y sus derechos, avanzando desde aquí en la construcción de la paz positiva de la que hemos venido hablando, sin embargo los estereotipos sociales aprobados están tan arraigados, que sigue siendo difícil la ruptura de estos paradigmas para la construcción de la cultura de paz.

A modo de conclusión vemos que existen normas aprobadas socialmente, que constituyen los géneros con una mirada subjetiva, donde se evidencian los parámetros específicos de las interacciones que pueden surgir para este tipo de construcciones, estando estas delimitadas por aspectos lineales, donde se encasillan las acciones que deben desempeñar hombres y mujeres, pero también es una invitación clara a la reconfiguración y resignificación de estas posturas por medio de narrativas positivas donde se rompan los cristales de estereotipos de ser y hacer para ellos y ellas.

Finalmente, en la categoría 3 La construcción de paz, como hemos evidenciado durante el proceso, va mucho más allá de las acciones planteadas por el Estado. Si bien es cierto Colombia es un estado social de derecho, el cual se propone fortalecer servicios y garantizar el ejercicio pleno de los derechos, que permiten mantener la calidad de vida de todos los habitantes de nuestro territorio, siendo miembros activos de la sociedad, también es relevante reflexionar sobre lo que como ciudadanos nos compete hacer, para apoyar las directrices estatales y propender por la vida de 
todos y todas en igualdad de condiciones. Las acciones diarias, nuestros comportamientos, la manera de expresarnos, pueden ser las formas más concretas de procurar el bienestar social. La construcción de un camino basado en valores, donde el otro sea un sujeto o sujeta de derechos igual y a la vez diferente reconociendo las particularidades y las diferencias que nos hacen ser quienes somos, se hace en los diferentes entornos en los que socializamos $y$ nos desenvolvemos.

Para construir ese camino es fundamental comprender cómo se hace la paz y qué tipo de paz estamos buscando fomentar. Como ya se mencionó en el capítulo conceptual la paz tiene varios significados, entre ellos la paz positiva y la paz negativa, vale la pena rescatar sus significados con el fin de soportar el análisis de esta categoría. La paz negativa es como se concebía hasta el siglo XX un estado de ausencia de guerra u otros tipos de acciones violentas. Es paz negativa en el sentido en que "proviene del hecho que se define en negativo, según lo que no es" (Ideas para construir la paz, diplomacia municipal y otras iniciativas de los gobiernos locales, 2008). En contraste tenemos la paz positiva la cual amplía el significado de paz encaminándola hacia la igualdad en las personas, la equidad social, el respeto en las relaciones, no solo interpersonales sino también con el entorno (medio ambiente), entonces se considera la paz como más que un estado, un proceso que puede mejorar constantemente las relaciones entre las personas y las condiciones estructurales. En ese sentido las acciones para la construcción de cultura de paz como mencionan los entrevistados inician propiamente en cada ser humano.

Tanto los entrevistados como los investigadores establecen acerca de las normas aprobadas socialmente, que constituyen los géneros con una mirada subjetiva, donde se evidencian los parámetros específicos de las interacciones que pueden surgir para este tipo de construcciones, estando estas delimitadas por aspectos lineales, donde se encasillan las acciones que deben desempeñar hombres y mujeres, pero también es una invitación clara a la reconfiguración y resignificación de estas posturas por medio de narrativas positivas donde se rompan los cristales de estereotipos de ser y hacer para ellos y ellas.

Los estudiantes convocados a esta reflexión analizaron desde sus experiencias cómo los discursos de igualdad de género en las universidades aportan a la construcción de paz, como menciona uno de los estudiantes "en la concepción de igualdad de géneros se puede llegar a entender la importancia de vivir en armonía, tener una conciencia verdadera de lo que es respetar, valorar y aceptar al otro tal y como es, a pesar de las diferencias que tenga. Todo esto nos enseña la igualdad: inclusión, equidad, tolerancia, aceptación, pero de nada sirve hacer campañas, encuestas y publicidad cuando en nuestro pensamiento eso no tiene algún grado de importancia" (estudiante entrevistado 1, 2016), desde aquí podemos evidenciar que existe conciencia frente al papel que tenemos como ciudadanos, en la ruptura de esquemas que no se alinean al surgimiento de una cultura de paz como forma de vida.

Desde estas miradas algunos docentes plantearon también, sus aportes a la construcción de paz desde sus roles de género en la universidad, en donde exaltaron "la importancia de una educación o reeducación de esta sociedad, que le permita hacer la diferencia, en donde la universidad aporte desde lo actitudinal, y nosotros en nuestro rol de maestros, nos demos a la tarea de posibilitar espacios de reconocimiento del otro, desde ejercicios que potencien un pensamiento crítico, generando compromisos que conlleven a acciones que permitan una transformación en nuestros paradigmas, proponiendo alternativas de solución, en primera medida de los problemas de la cotidianidad que pueden surgir en el ámbito universitario, lo que les permitirá unas bases más sólidas que fortalezcan su pensamiento crítico para hacer parte de una construcción de paz a nivel social" (Docente entrevistado, 2016).

Por su parte las directivas se asumen como parte del cambio; una de ellas que plantea "Primeramente las motivaciones que me impulsan a involucrarme en la promoción y defensa de los derechos humanos de las mujeres y la diversidad sexual, son debido a mi experiencia en el hogar, en la academia, en los espacios públicos y en el sector laboral; debido a estos ámbitos me percaté 
que cotidianamente existen, persisten y se recrean distintas violencias hacia las mujeres y las personas que tienen diversidades sexuales en Colombia. Desde desmeritar las acciones de mujeres y nuevas masculinidades y feminidades $e$ inculcar formas de ser, sentirnos temerosas $y$ temerosos de habituar algunos espacios públicos pensando en que probablemente podemos ser agredidas y agredidos de distintas formas desde el "piropo", las vallas publicitarias y los ataques físicos y sexuales, hasta tener que sublevarnos para que nuestra opinión y posición sea valorada de la misma manera que la de los compañeros de academia y trabajo. Además, considero que sí las colombianas y los colombianos contempláramos la significativa labor de las mujeres y las nuevas feminidades y masculinidades, se podría concebir un país en paz con justicia social; luego se repensaría una sociedad que históricamente se ha proyectado como patriarcal y que hasta el día de hoy ello ha sido resultado de un país en guerra, con hambre y desigualdad. Por lo que mi aporte a la construcción de paz desde mi identidad de género, es generar espacios de conocimiento, discusión y reflexión con acciones directas como aportar, gestionar y ser parte de procesos organizativos que se proyectan desde la equidad de género y el empoderamiento de las mujeres, reconociéndonos como un sector poblacional subordinado, debido a una herencia histórica moral, social y cultural, además de tratar en mi diario vivir concebirme como una mujer empoderada que le apuesta a la diversidad sexual y la otredad"(directiva entrevistada, 2016).

Es necesario construir una propuesta política que se proyecte a lo educativo desde una comprensión social y crítica, donde se evidencie el reconocimiento de la multiplicidad, en un diálogo que trascienda las relaciones de poder y se encamine a la equidad de conocimientos y saberes de todos y todas, sobre la base de las experiencias; la transformación social debe implicar una perspectiva que permita vislumbrar la complejidad de las comunidades, entendiendo las nuevas reconstrucciones y redistribuciones de nuestros roles en todos los ámbitos. Afianzar los procesos incluyentes en los espacios académicos, donde no solo se empodere a las mujeres sino, donde otras poblaciones históricamente relegadas puedan ser activas, propositivas y constructoras de nuevos órdenes en el sistema de nuestra sociedad. Es importante ir más allá de la moda, hablar de inclusión no debe quedarse en el discurso debe ser fundamentada y transformadora, trascender esos imaginarios que se producen, se naturalizan y normalizan por medio de los diálogos cotidianos que vivenciamos, que configuran los conceptos y comportamientos de lo que debe ser un hombre y una mujer, llevándolos a la adaptación de normativas tradicionalistas, determinando la idealización jerárquica de roles sociales. Por esto la universidad debe procurar romper los esquemas esencialistas de hombres superiores y dominantes y mujeres con habilidades para el hogar y el cuidado del otro, esto desde la apertura de campos de investigación que impliquen una postura más flexible frente a las personas, las prácticas, los saberes y los valores.

Es necesario resaltar en este punto desde la mirada de la complejidad, es importante analizar el conflicto a la luz del principio dialógico de esta perspectiva entendiendo que:

a diferencia de la visión convencional simplificadora y fragmentada sobre la relación entre orden y desorden, la visión compleja e integral permite comprender el desorden y aceptarlo tal como es: algo normal, inevitable e imprescindible; sin lo que no habría movimiento, innovación o vida. De modo que es positivo y debe ser aprovechado para transformar realidades. Con el conflicto ocurre igual, también se ha reputado como malo, negativo, algo aberrante que se debe evitar o eliminar (...). (Aristizabal, 2003, p. 175)

Al hablar de conflicto nos encontramos con herencias de este término que lo reprueban y encasillan en lo malo, relacionándolo directamente con la violencia. Sin embargo, el conflicto debe analizarse a la luz de dos posturas, desde una argumentación epistemológica, probabilidades e improbabilidades, que son directamente proporcionales a la perspectiva del principio de incertidumbre y el hologramático, entendiendo que el todo y las partes se retroalimentan y que no se mueven linealmente, sino que las situaciones son inciertas, haciendo la realidad poco previsible y contingente por esto nuevas realidades son posibles. Desde allí, es 
importante tener en cuenta las relaciones que se desarrollan en los sistemas sociales y todo lo que implica reconocer las correlaciones entre las diversas culturas que hacen parte de ellos y sus implicaciones, ya que estas nuevas ideas particulares y novedosas dan lugar a la diversidad de construcciones, que surgen en un exceso de orden o desorden, lo que nos lleva a reconfigurar el sistema en una nueva organización. Es necesario reflexionar que el conflicto considerado como desorden, comprende el surgimiento de competencias, concurrencias, rivalidades, en lo social, lo político y lo económico, enfrentamientos que son inherentes a las relaciones sociales, situaciones que deben ser resueltas, transformadas, concertadas y negociadas. Es así como el conflicto contribuye al dinamismo, al movimiento y a la regeneración permanente de los procesos sociales. La resolución de los conflictos, en la reflexión de los intereses comunes, las complementariedades y las interdependencias, permiten un nuevo orden, sin embargo, la omisión de estas características en un conflicto y la superposición del antagonismo y la competencia, redundan en la agudización del conflicto y por ende surgen la agresión y la violencia. Por esta razón el conflicto debe verse más allá de la mirada lineal y fragmentada como lo negativo, para ser visto desde la multiplicidad de causas y efectos que surgen de una situación conflictiva, que permiten la construcción colectiva de las nuevas realidades.

\section{Conclusiones}

Teniendo presente el objetivo general de esta investigación, podemos concluir que, este proceso nos permitió comprender que las relaciones de género, condicionan la construcción de la cultura de paz en un contexto como la universidad, ya que educar para la igualdad y la equidad, desarrollando planes destinados a eliminar el concepto de discriminación en las relaciones, que surgen en las escuelas de educación superior, en donde los agentes educativos juegan un papel importante por medio de comunicaciones interpersonales y de la aceptación de las diferencias, que parten de su capacidad de permitir y diseñar nuevas propuestas que inviten a evitar las desigualdades, consolida la perspectiva de una sociedad más justa, entrelazando pensamientos, sentires y el hacer, que nos permitan apostarle a la paz, como un derecho que se construye entre todos y todas. No como la ausencia de guerra sino como una condición de tranquilidad, plenitud y calidad de vida.

Son múltiples las percepciones que surgieron de la interacción con los entrevistados, son valiosas las posturas y reflexiones que cada uno hace desde sus vivencias y desde sus realidades, en cada una de las universidades participantes, es necesario exaltar a modo de conclusión el papel fundamental que tienen las universidades, en la formación y transformación de valores que impactan en la construcción de la cultura de paz con enfoque de género, puesto que al reconocer la responsabilidad de las escuelas de formación superior, como uno de los espacios principales en la formación de futuros constructores $y$ constructoras de sociedad, siempre deben contemplar una convivencia de calidad, en donde se viva de manera clara la igualdad, donde se diseñen espacios académicos que posibiliten condiciones necesarias para lograr aprendizajes significativos, que aporten al enriquecimiento de la diversidad y el respeto. Las posturas de los estudiantes, docentes y directivos de los tres claustros educativos participantes refieren que la cultura de paz, debe velar por las formas de actuar de los agentes que hacen parte de la misma comunidad educativa, fortalecer los espacios de diálogo y participación en los que todos los miembros hagan parte de la construcción colectiva de la garantía de derechos y sean un todo caminando hacia un objetivo, la paz.

Surgen reflexiones desde una perspectiva educativa, en donde hay una necesidad de transmitir mensajes de cultura de paz: interlocución de experiencias de convivencia, entre universidades y en otros ámbitos sociales, dando a conocer iniciativas que se quieren realizar y fomentar. Desde las universidades se evidencia como un paso importante en la búsqueda de un lugar más pacífico, la proyección de un mundo configurado en un propósito de paz, otro aspecto importante a implementar son los mensajes de cultura de paz, que se pueden transmitir incitando y sensibilizando a la comunidad educativa en general, a participar en 
campañas de construcción de paz: desde una apropiación más significativa y reflexiva, de lo que realmente esto significa en nuestra sociedad.

Finalmente, se concluye que al problematizar la importancia del tema del género y las relaciones en un contexto de formación superior, se puede pensar en un proceso educativo desde la corresponsabilidad de toda la comunidad educativa y la sociedad en general, teniendo en cuenta las familias, los ámbitos laborales y los entornos en los que confluyen las comunidades y sus miembros, ya que estos son espacios de socialización, que permiten compensar las relaciones y enfocar a la ruptura de imaginarios y prejuicios que inciden en el desarrollo y fortalecimiento de las tramas relacionales que allí se entretejen.

\section{Recomendaciones}

Hay varias acciones que deben replantearse en los modelos educativos implementados en las universidades en nuestro país, pasando de la formación académica exclusivamente, a crear escenarios en los que la investigación social, la creación de políticas internas y la articulación interinstitucional, promuevan la formación de personas y profesionales capaces de construir nuevas realidades y modelizar en los entornos con otros grupos sociales, la Cultura de Paz real en Colombia.

pues más que a aprender números y letras a la escuela se ha ido más de un siglo y aun se va a experimentar el entramado de normalización/moralización/penalización de conductas. Un entramado que admite y depura también las diferencias de clase y de género (Jesús Martín Barbero). (Fernandez, Hernandez \& Paniagua, 2005, p. 76)

Es claro que la universidad es un espacio de formación, al que no todos los colombianos tienen acceso, tristemente es un derecho que es privilegio de pocos. Sin embargo, es necesario pensar la universidad como un sistema que permea continuamente otros sistemas sociales, puesto que es una comunidad educativa en la que el análisis cultural y teórico está presente en todos sus espacios. La multiculturalidad presente en la universidad y la diversidad de saberes que allí confluyen, demandan del alma mater la creación, fortalecimiento y reconocimiento de espacios de interacción, no solo entre estudiantes sino también con la participación de docentes y directivos que se involucren en la visibilización y formación, en la relevancia de los valores, actitudes y aptitudes e incluso de pautas basadas en la equidad y en la no existencia de diferencias sobre las relaciones que deben darse entre hombres y mujeres que interactúan diariamente. La construcción de estas relaciones sólidas concluye en la construcción de una cultura universitaria que inevitablemente impacta otros entornos sociales en los que se desenvuelven los y las jóvenes.

Como hemos visto, existe en la percepción de los entrevistados y en general en la comunidad, una idea de la evolución que hemos tenido en la construcción de entornos sociales más equitativos, en cuanto a perspectiva de género, desde la mirada de las facultades humanas y sociales, pero es evidente que aún existen situaciones que son entendidas como violencia de género y que socialmente son validadas $y$ pasan desapercibidas en las comunidades, situaciones que se hace necesario visibilizar para romper dichos roles y estereotipos relacionales que aperturen una cultura de paz tangible.

La paz es una mujer que trabaja por construir una mejor sociedad a partir del ejercicio de la autonomía, para aportar desde su libertad la capacidad de amar, crear y vivir. Gisselle Cifuentes-Líder feminista

Este artículo, hace un llamado a romper las cadenas de estigmas y paradigmas sociales y culturales de los roles de género, es un llamado a las mujeres a despertar, a luchar, a exigir y sobre todo a trabajar en el nuevo lenguaje de poder sobre la formación de nuevos sentires y pensamientos y a los hombres a desligarse del molde estereotipado impuesto. En este sentido, es importante que la sociedad civil comprenda que el conflicto armado va más allá de las diferencias políticas y la lucha por el poder a fuerza y violencia, el conflicto surge de las relaciones dadas en los distintos entornos, desde el hogar en lo privado, hasta lo social en lo público. Desde allí es importante revisar el papel que tiene la mujer en los procesos de paz nacionales, regionales y locales; es sabido que 
hay una deuda histórica frente a la participación de la mujer en la sociedad y frente a los imaginarios de comportamientos y de roles que han mantenido a la mujer en el olvido y el silencio, siendo la principal afectada por las acciones violentas y la actriz principal, en un proceso de cambio cultural, puesto que la mujer tiene un desempeño importante en el desarrollo social de sus comunidades, en la construcción de nuevas dinámicas y las transformaciones de las realidades y narrativas propias de las colectividades.

Las mujeres colombianas han tenido que salir de sus tierras, dejando costumbres, historias y raíces, han sido las huérfanas de los padres que la violencia se ha llevado, viudas de los esposos y compañeros que han desaparecido y madres que lloran la ausencia de sus hijos que nunca más vuelven a ver, pero que no pierden la esperanza del volver a empezar, porque la capacidad resiliente de las mujeres es de tal nivel, que en medio del desplazamiento forzado, las agresiones de las que son víctimas en casa o en la calle, la discriminación que sufren en algunos casos en entornos académicos y laborales, se hacen de nuevos saberes, comprenden nuevas realidades, se abren como flores a las nuevas circunstancias y crean expectativas para la vida en medio de la muerte, para finalmente asumir las responsabilidades de los nuevos roles, en la nueva vida. El cese de las confrontaciones no garantizará la paz, la firma de un acuerdo será un documento cuyo real impacto se dará, si y solo si, entendemos que el proceso de post acuerdo debe planificarse bien, partiendo de la necesidad de construir una realidad distinta, que traiga consigo dinámicas sociales y educativas nuevas, en las que recomposición, conciliación, reconciliación, reparación, inclusión y respeto por cada uno, se vuelvan parte de la cotidianidad, no solo desde la verbalidad, sino también en la realidad, esto, de la mano de mujeres capaces de curar, cuidar, cultivar, construir, convocar, contener, convencer, concertar, culturizar, de empoderar y empoderarse y ser autónomas en sus vidas.

El papel de la mujer en la conciliación y la construcción de paz debe ser el punto de partida para que esta nueva cultura sea duradera y permita garantizar la sostenibilidad del desarrollo; El empoderamiento ha sido considerado como una de las vías fundamentales para el desarrollo y la transformación de comunidades (Montero, 2003) puesto que desde allí se evita la re victimización y la culpabilización centrándose en la proposición de acciones pertinentes y eficaces que permitan el mejoramiento de la calidad de vida y la integración a una vida social activa desde las familias.

Es también, un llamado a construir de la mano, hombres y mujeres, nuevas formas de ver y hacer el mundo, nuevas oportunidades de hacer ciudadanía basados en rescatar valores y virtudes la cultura femenina que les han sido negadas a los hombres. Es la forma de retar social y culturalmente a la sociedad comprendiendo que es complejo, puesto que la transformación en lo cultural a nivel institucional, debe ser profunda para desarraigar el valor machista impuesto en nuestra forma de ver el mundo. Esta es la oportunidad de ir más allá de los protocolos y los acuerdos firmados en papel es la oportunidad de hombres y mujeres de propender por la equidad para todos y todas en las diferentes esferas de la sociedad, transformando la masculinidad a masculinidades diferenciándola del dominio, de la agresión, de la violencia, la indiferencia y la supresión de las emociones y los afectos. Es la oportunidad de centrarnos en las principales víctimas de las violencias simbólicas y estructurales niños y niñas quienes desde el lenguaje reproducen los estereotipos, son víctimas del patrón patriarcal que busca endurecer las opciones expresivas de niños y hombres promoviendo la omisión de muchas de las dimensiones del ser humano, es desde aquí desde donde rompemos el modelo hegemónico de masculinidad el cual promueve el maltrato y la deshumanización. Y como menciona Javier Omar Ruiz:

Modificar estas pautas de crianza y de socialización es una tarea urgente. El trabajo de modificación tiene múltiples puertas de entrada, una de ellas, la vía afectiva, es altamente movilizadora por cuanto reta en su máximo punto débil, a toda la estructura patriarcal. La ternura puede tener el tamaño del cuerpo y el afecto la edad de la vida. El género es absolutamente secundario. (Ruiz, 2000) 


\section{Referencias}

Aristizábal, J. (2003). El conflicto y su transformación desde el pensamiento complejo. Estudios políticos No 23, 171- 180.

Calderón, P. (2009). Teoría de conflictos de Johan Galtung. Revista de paz y conflictos, 60-81.

Cárdenas, R., Rivera, M. y Rivas, J. (2004). La teoría de la complejidad y su influencia en la escuela. Revista de Teoría y Didáctica de las ciencias sociales. Número 9 enero- diciembre. http://www.redalyc.org/pdf/652/65200908.pdf

Martínez., C y Mirón, M. (2000). La paz desde la perspectiva de los estudios de género: una aportación fundamental para construir un mundo más igualitario, justo y pacífico. En M. \&. Mirón. España: Universidad de Granada.

Escámez, J. (2004). La Educación para la Promoción de los Derechos Humanos de la Tercera Generación. Universidad de Valencia, España, 81- 100.

Espinosa, R. (2007). La violencia en la modernidad, hacia una alternativa de paz. México D.F.

Expósito, F. (2011). Violencia de género. La asimetría social en las relaciones entre mujeres y hombres favorece la violencia de género. Granada, España: Mente y Cerebro.

Fernández, H. y Paniagua. (2005). Violencias de género en la universidad de Antioquia Medellín: Lealon.

Fisas, V. (1998). Una Cultura de Paz. En V. Fisas, Cultura de paz y gestión de conflictos. Barcelona: Icaria/NESCO.

Gallardo, E. (12 de junio de 2014). Atlas.ti qualitative data analysis. Obtenido de https://atlastiblog.wordpress.com/2014/06/12/utilizacion-del-programa-de-analisis-cualitativoatlas-ti-para-gestionar-y-analizar-datos/

García, B. E. (2000). Identidad y Alteridades. Mérida, Venezuela: Universidad de los Andes.

García, C. (2000). Masculino/Femenino ¿Y yo? identidad o identidades de género.

González L. (s.f.). (1998) Entre la realidad y la utopía. Curso de Moral. Santander.

Herrera, J. D. (2012). Horizonte Hermenéutico de las Ciencias Sociales. En J. D. Herrera, La comprensión de lo social. Académica Española.

Diputación de Barcelona, I’ Escola de Cultura de Pau. Ideas para construir la paz, diplomacia municipal y otras iniciativas de los gobiernos locales. Monografía (2008). Barcelona.

Bastidas, J. y Hernández, R. (2008). Género y Educación para la Paz: Tejiendo Utopías posibles. Revista Venezolana de estudios de la mujer.

Jiménez, F. (2006). Paz neutra y la realidad indígena como constructora de espacios neutrales de paz. Red de Revistas Científicas de América Latina, el Caribe, España y Portugal, 355,356.

Jiménez, M. J. (2012). Breve estudio sobre las ideas de paz. Bogotá: Observatorio para la Paz.

Juárez, J. y Comboni, S. (2012). Epistemología del pensamiento complejo. Reencuentro, 39.

Lagarde, M. (1990). Identidad Femenina.

Lamas, M. (2000). Diferencias de sexo, género y diferencia sexual. Cuicuilco, vol. 7, núm. 18.

Lederach, J. P. (s.f.). (2017). ¿Qué es educar y formar para la paz y cómo hacerlo? Página 43-70.

Loaiza, A. M. (2011). Investigación para la paz y perspectivas de género: Desvelando las relaciones pacíficas entre hombres y mujeres. Eleuthera.

López, M. (2006). Política sin violencia, La no violencia como humanización de la política. UNIMINUTO, 106.

López, M. C. (1994). Educación para la paz y formación de profesores. Universidad de Granada, 317-348.

Bolaños, L. y Jiménez, R. (2007). La formación del profesorado en Género. Revista de Investigación Educativa Vol. 25, 77-95.

Martínez M. La investigación cualitativa etnográfica en educación. México: Trillas; 1998. p. 65-68.

Magallón, C. (1993). El sistema sexo- género y sus implicaciones para la paz. Página 61-76.

Magallón, C. (2005). Epistemología y Violencia aproximación a una visión integral sobre la violencia hacia. Fundación Seminario de Investigación para la Paz de Zaragoza, 1-17.

Magallón, C. (2006). Mujeres en pie de paz: pensamiento y prácticas. Siglo XXI. 
Molina, F. M. (25 de junio de 2009). Una cultura de paz compleja y conflictiva. Obtenido de Revista de Paz y Conflictos: http://revistaseug.ugr.es/index.php/revpaz/article/view/441/494.

Morín, E. (febrero de 2003). Gazeta de Antropología. Obtenido de http://www.ugr.es/ pwlac/G20_02Edgar_Morin.html.

Muñoz, $\quad$ F. $\quad$ (2006). La $\quad$ Paz imperfecta. Instituto de la Paz y los Conflictos de la Universidad de Granada (España).

Naciones Unidas de Nueva York ONU. (1996). Informe de la Cuarta Conferencia Mundial sobre la Mujer.

Pannikkar, R. (1993). Paz y desarme cultural. Santander.

Parra, D. y Tortosa. J. (2003). Violencia estructural: una ilustración del concepto. Grupo de Estudios de Paz y Desarrollo, Universidad de Alicante.

Peláez, A., Rodríguez, J., Ramírez, S., Pérez, L., Vázquez A., y González, L. (s.f.). (2008) Obtenido de https://www.uam.es/personal_pdi/stmaria/jmurillo/InvestigacionEE/Presentaciones/Curso_10/ Entrevista.pdf

Romero, F. (2009). Violencia y Cultura de Paz. Aportes Andinos № 25.

Ruiz, J. O. (2000). Los niños están siendo víctimas de la hombría. Colectivo Hombres y Masculinidades.

Suarez, Z. (2011). Educar para la paz: Tema urgente en nuestra sociedad. Revista Ensayo Pedagógicos Vol. $1,71-82$.

Tarride, M. (1996). Complejidad y sistemas complejos. Manguinhos.

Yudkin, A. (2014). Educar para la convivencia escolar y la paz: Principio y prácticas de esperanza y acción compartida. Ra Ximhai Vol. 10. 